\title{
An audit of patients with abruptio placentae in the Pretoria Academic Complex
}

\author{
B Magagula, ${ }^{1} \mathrm{MB} \mathrm{ChB}, \mathrm{T}$ Nkwenika, ${ }^{2} \mathrm{BSc}, \mathrm{S}$ Adam, ${ }^{1} \mathrm{PhD}$ \\ ${ }^{1}$ Department of Obstetrics and Gynaecology, Faculty of Health Sciences, University of Pretoria, South Africa \\ ${ }^{2}$ Biostatistic Research Unit, South African Medical Research Unit, Pretoria, South Africa
}

Corresponding author: S Adam (sumaiya.adam@up.ac.za)

Background. Obstetric hemorrhage is the third most common cause of maternal death in South Africa. Abruptio placentae (AP) contributed to $12.9 \%$ of deaths caused by haemorrhage between 2017 and 2018.

Objective. To investigate the risk factors, management and outcomes of pregnancies affected by AP.

Methods. We conducted a prospective descriptive study in a one-year period at the Pretoria Academic Complex (PAC). All patients who presented with AP were recruited for the study. The demographics, risk factors, management, complications and outcomes were reported using descriptive statistics.

Results. One hundred women with AP presented during the study period, representing an incidence of $1 \%$ of pregnancies at PAC. Sixtyone percent of the women had caesarean delivery while $37 \%$ had vaginal delivery. A minority of women presented with known risk factors of AP in this setting. Two comparison groups were defined based on the degree of placental separation from the decidua, i.e. $>50 \%$ and $<50 \%$. A woody hard abdomen occurred more frequently in patients with $>50 \%$ AP ( $43 \%$ v. $57 \%$; $p=0.002)$. More women had postpartum haemorrhage in the $>50 \%$ group $(67 \%$ v. $33 \% ; p=0.001)$. Fifty-eight $(58 \%)$ live babies and $44(44 \%)$ stillborn babies were delivered.

Conclusion. The known risk factors of AP were largely absent in women with AP in this setting. The typical clinical findings of AP were late signs associated with severe placental separation. Early resuscitation and delivery of the fetus can positively influence perinatal and maternal morbidity and mortality.

S Afr J Obstet Gynaecol 2019;25(3):79-83 https://doi.org/10.1796/SAJOG.2019.v25i3.1482

Abruptio placentae (AP) is defined as premature separation of a normally located placenta before delivery of the fetus. ${ }^{[1]}$ The incidence of AP is reported to be $0.8-1 \%$ of all pregnancies. ${ }^{[2]}$ This condition remains a significant cause of perinatal morbidity and mortality. A study in the USA reported a perinatal mortality of 110/1 000 births associated with AP compared with 8.2/1 000 in all other births. ${ }^{[3]}$ Obstetric haemorrhage is the third most common cause of maternal death in South Africa (SA). AP was found to contribute $12.9 \%$ to deaths caused by haemorrhage in the last triennium according to the 2014 - 2016 Saving Mothers Report. ${ }^{[4]}$

The aetiology of AP is not fully understood; however, various risk factors have been found to be associated with the condition. It is postulated that AP is an acute event that is a culmination of chronic placental pathology. ${ }^{[5]}$ Risk factors include hypertension, chorioamnionitis, rupture of membranes, polyhydramnios, advanced maternal age, intrauterine growth restriction, multiparity, retroplacental myomas, sudden decompression of the uterus, alcohol, smoking, previous history of AP, trauma, and uterine manipulation (external cephalic version)..$^{[5-9]}$

The pathophysiology of AP includes bleeding at the utero-placental interface. ${ }^{[5,10]} \mathrm{A}$ combination of acute and chronic processes increases the risk of $\mathrm{AP}^{[10]}$ The haematoma may be small and self-limiting in women with mild AP. ${ }^{[9,10]}$ A growing haematoma dissects the placenta off the uterine decidua. The larger the separation from the uterine wall, the higher the morbidity for the mother and the fetus. ${ }^{[10,11]}$
AP is usually diagnosed clinically. Symptoms and signs include abdominal pain, back pain, vaginal bleeding, tetanic contractions, and a non-reassuring fetal condition. ${ }^{[1]}$ When the separation is more than $50 \%$ of the placental attachment to the decidua, fetal death and disseminated intravascular coagulopathy are common. ${ }^{[12]}$ The attending doctor estimates the percentage of AP by examining the clot expelled relative to the placenta and examining the placenta attachment at caesarean delivery. ${ }^{[1,11,12]}$ Adverse outcomes of abruptio placentae can be categorised into fetal, maternal and neonatal. Fetal outcomes include intrauterine growth restriction, fetal distress and intrauterine fetal death. Maternal outcomes include anaemia, hypovolaemia/shock, coagulopathy, renal failure, hysterectomy, need for blood transfusion, and death. Neonatal complications include preterm birth, neonatal death, and small-for-gestational age. ${ }^{[1]}$

The management of AP is aimed primarily at ensuring the wellbeing of the mother and secondarily that of the fetus. Resuscitation is initiated without delay, and it requires the administration of intravenous fluid and blood products. Continuous monitoring of maternal and fetal well-being is essential. Vaginal delivery for $\mathrm{AP}$ with a demised fetus is ideal, as it is associated with the lowest risk for adverse outcomes in the mother. If the fetus is alive or the mother is haemodynamically unstable and delivery is not imminent, it is prudent to consider caesarean delivery. ${ }^{[13]}$

Current teaching on AP still refers to risk factors and protocol management. However, there are limited data on risk factors and 
outcomes of AP in SA, especially in recent years. In addition, the protocol prescribes the use of 4 units of red packed cells and 4 units of fresh-frozen plasma; ${ }^{[1,11]}$ however, blood products are an expensive and scarce resource and therefore not readily available in all clinical settings.

The aim of the study was to evaluate the most common risk factors for AP at our centre, audit our management of AP (including the use of blood products), and report on fetal and maternal outcomes.

\section{Methods}

This was a prospective descriptive study of all women presenting with AP managed from 1 December 2017 to 30 November 2018 at the Pretoria Academic Complex (PAC), which comprises the Kalafong Provincial Tertiary Hospital (KPTH) and Steve Biko Academic Hospital (SBAH). These are two tertiary hospitals that serve the greater Tshwane area. Midwife-led obstetric units and district hospitals refer patients to these two hospitals. All patients with AP are referred to a tertiary hospital in this setting. There are on-site laboratories and blood banks available at both hospitals.

All patients managed with AP during the study period were invited to be included in the study and were recruited following informed consent. The study received approval from the Health Sciences Research Ethics Committee of the University of Pretoria (ref. no. 416/2017). A pregnancy was diagnosed with AP if a retroplacental clot was diagnosed at delivery in a patient who presented with typical signs and symptoms (abdominal pain, tetanic contractions, fetal distress, antepartum haemorrhage). Pregnancies $\geq 26$ weeks' gestation as determined by the consulting obstetrician were included in the study, as this is the limit of viability at SBAH and KPTH. The principal investigator recruited patients with AP, as identified by the obstetric registrars on call.

AP was classified according to clinical features shown in Table $1{ }^{[14]}$ and the attending obstetric registrar estimated the macroscopic placental separation which was recorded as percentages. Data were retrieved from patient records and the women were interviewed to complete any missing information. Demographic factors, presenting symptoms and signs, management and outcomes were recorded on a data collection questionnaire. The patients were recruited on admission following stabilisation. They were interviewed again prior to delivery (i.e. between 3 - 7 days).
Data were analysed using SPSS (IBM Corp., USA). Descriptive statistics in the case of normally distributed data consisted of means, standard deviations, and medians and ranges if the data were not normally distributed. Categorical data were summarised using frequencies and percentages, $p<0.005$.

\section{Results}

Of the 9911 women presenting to the KPTH and SBAH, the mean age was 28.7 years (range $17-55$ ) and the overall incidence of AP was $1 \%(n=100 / 9$ 911). Forty-two presented to KPTH and 58 were seen at SBAH. Table 2 shows the demographic characteristics and risk factors of the study population. Of the 100 women, $7 \%$ had class $0,47 \%$ had class $1,4 \%$ had class 2 , and $42 \%$ had class 3 AP.

Eighty-six percent of the women had received antenatal care, $88 \%$ had a haemoglobin level $\geq 10 \mathrm{~g} / \mathrm{dL}$, and $36 \%$ had hypertensive disorders during pregnancy. Of the women with hypertensive disorders, 14\% $(n=5 / 36)$ had chronic hypertension, 5 (14\%) had gestational hypertension and $72 \%(n=26 / 36)$ had pre-eclampsia. One (1\%) woman reported having used cocaine.

Table 3 shows the presentation and management of the study population. One (1\%) woman had AP that was diagnosed at an early gestational age (26 weeks) and was managed conservatively, and one (1\%) case had a history of AP in a previous pregnancy. Of the 100 women with AP, 59 had $<50 \%$ AP and 39 had $>50 \%$ AP.

Fifty-three women were normotensive with blood pressure (BP) readings of between 90/60 and 140/90 $\mathrm{mmHg}$ at presentation and 6 women were in hypovolaemic shock with BP measurements $<90 / 60$ $\mathrm{mmHg}$. Twenty-one women presented with BP between 140/90 and $159 / 109 \mathrm{mmHg}$, and 20 had BP readings $>160 / 110 \mathrm{mmHg}$.

Fifty-eight live babies were delivered, 60 women had live fetuses on admission, and 39 women presented with an intrauterine fetal demise. One fetus was alive on admission, but died within an hour, prior to delivery.

Forty-four (76\%) of the live babies were in the $<50 \%$ AP group compared with $14(24 \%)$ of the live babies found in the $>50 \%$ AP group. Thirty-six (82\%) babies in the $<50 \%$ AP group had a 5-minute Apgar score $>7$, compared with $8(18 \%)$ in the $>50 \%$ AP group. There were 42 (42\%) stillborn babies delivered. Of the stillbirths, $33(78 \%)$ were fresh stillbirths (FSB) while $9(22 \%)$ were macerated stillbirths (MSB). Eight (89\%) of the MSBs were in the $>50 \%$ AP group while $1(11 \%)$ was in the $<50 \%$ AP group.

Table 1. Classification of abruptio placentae

\begin{tabular}{ll}
\hline Class & Clinical features \\
\hline 0: Asymptomatic & Discovery of a blood clot on the maternal side of a delivered placenta \\
& Diagnosis is made retrospectively \\
1: Mild & No sign of vaginal bleeding or a small amount of vaginal bleeding \\
& Slight uterine tenderness \\
& Maternal blood pressure and heart rate within normal limits \\
& No sign of fetal distress \\
& No sign of vaginal bleeding to moderate amount of vaginal bleeding \\
2: Moderate & Significant uterine tenderness with tetanic contractions \\
& Change in vital signs: maternal tachycardia, orthostatic changes in blood pressure \\
& Evidence of fetal distress \\
& Clotting profile alteration: hypofibrinogenaemia \\
& No sign of vaginal bleeding to heavy vaginal bleeding \\
3: Severe & Tetanic uterus/ board-like consistency on palpation \\
& Maternal shock \\
& Clotting profile alteration: hypofibrinogenaemia and coagulopathy \\
& Fetal death
\end{tabular}


Table 2. Description of the study population $(N=100)$

\begin{tabular}{|c|c|}
\hline Variable & $n(\%)$ \\
\hline \multicolumn{2}{|l|}{ Age (years) } \\
\hline$\leq 19$ & $4(4)$ \\
\hline $20-34$ & $78(78)$ \\
\hline$\geq 35$ & $18(18)$ \\
\hline \multicolumn{2}{|l|}{ Parity } \\
\hline Nulliparous & $27(27)$ \\
\hline $1-2$ & $59(59)$ \\
\hline$\geq 3$ & $14(14)$ \\
\hline \multicolumn{2}{|l|}{ HIV reactive } \\
\hline Yes & $14(14)$ \\
\hline No & $86(86)$ \\
\hline \multicolumn{2}{|c|}{ Cigarette smoking } \\
\hline Yes & $5(5)$ \\
\hline No & $95(95)$ \\
\hline \multicolumn{2}{|c|}{ Other substance use } \\
\hline Yes & $1(1)$ \\
\hline No & 99 (99) \\
\hline \multicolumn{2}{|l|}{ Antenatal care } \\
\hline Yes & $86(86)$ \\
\hline No & $14(14)$ \\
\hline \multicolumn{2}{|c|}{ Anaemia in pregnancy } \\
\hline Yes & $88(88)$ \\
\hline No & $12(12)$ \\
\hline \multicolumn{2}{|l|}{ High BMI } \\
\hline Yes & $1(1)$ \\
\hline No & $99(99)$ \\
\hline \multicolumn{2}{|c|}{ Chronic hypertension } \\
\hline Yes & $5(5)$ \\
\hline No & $95(95)$ \\
\hline \multicolumn{2}{|c|}{ Gestational hypertension } \\
\hline Yes & $5(5)$ \\
\hline No & $95(95)$ \\
\hline \multicolumn{2}{|l|}{ Pre-eclampsia } \\
\hline Yes & $26(26)$ \\
\hline No & $72(72)$ \\
\hline \multicolumn{2}{|c|}{ Antepartum haemorrhage of unknown origin } \\
\hline Yes & $2(2)$ \\
\hline No & $98(98)$ \\
\hline \multicolumn{2}{|l|}{ Chronic AP } \\
\hline Yes & $1(1)$ \\
\hline No & 99 (99) \\
\hline \multicolumn{2}{|l|}{ Previous AP } \\
\hline Yes & $1(1)$ \\
\hline No & 99 (99) \\
\hline
\end{tabular}

We found a stillbirth rate of $420 / 1000$ deliveries for our study population.

The timeframe from presentation to delivery was $<1$ hour in 35 (35\%) women, $1-3$ hours in 33 (33\%) women, $3-7$ hours in 21 (21\%) women and $>7$ hours in $11(11 \%)$ women. The average time from presentation to delivery was 90 minutes in women presenting with live fetuses and 240 minutes in women presenting with intrauterine fetal demise.

Table 4 shows the maternal and fetal outcomes of the study population. While there were no maternal deaths to AP in the 100 women during the study period, 8 women had acute kidney injury, 1 had disseminated intravascular coagulation, and 17 had isolated thrombocytopaenia. These acute abnormalities were corrected prior to discharge in all patients.

Forty-three (43\%) women experienced a primary postpartum haemorrhage (PPH), which was managed medically with uterotonics in 33 (76.7\%) of these women. Seven (17.1\%) women with PPH were managed surgically with a B-Lynch suture which was successful in 6 women. Two women had a hysterectomy, one of which was following an unsuccessful B-Lynch suture. Balloon intra-uterine tamponade was not used in any of the patients.

\section{Discussion}

The incidence of AP in this study (1\%) is similar to what is reported in the literature. ${ }^{[2]}$ The well-documented risk factors of AP such as hypertension, smoking, and absence of antenatal care was present in a small minority of patients with AP in this setting. Few patients in our study had risk factors for AP. Our study population consisted of a majority of women who were non-smokers, attended antenatal care, had a normal BMI, had no previous history of AP and were HIV nonreactive. In an SA study, Brink et al. ${ }^{[12]}$ found an increased risk of AP in patients who smoked cigarettes, were unbooked antenatally, and had hypertension. Similarly, a study in Nigeria ${ }^{[15]}$ found that $83.7 \%$ unbooked patients developed AP, and Boisramé et al..$^{[8]}$ found an increased risk of AP in patients who had hypertensive disorders in pregnancy and a parity $\geq 3$.

Patients who had $<50 \%$ AP had abdominal pain and vaginal bleeding as a more common complaint than those who had $>50 \%$ AP, although this was not statistically significant. Patients with $>50 \%$ AP reported absent or reduced fetal movements more frequently than patients with $<50 \%$ AP. This is therefore a late symptom of AP, as outcomes are poorer for this group of patients. As reported in the

Table 3. Presentation and management of patients with AP

\begin{tabular}{|c|c|c|c|c|}
\hline Variable & $<50 \%$ AP $(N=39), n(\%)$ & $>50 \%$ AP $(N=59), n(\%)$ & Total $(N=98)^{*}$ & $p$-value \\
\hline Abdominal pain & $46(64)$ & $26(36)$ & $72(73.5)$ & 0.215 \\
\hline Vaginal bleeding & $40(59)$ & $28(41)$ & $68(69.4)$ & 0.674 \\
\hline Reduced/absent fetal movement & $11(41)$ & $16(59)$ & $27(27.6)$ & 0.015 \\
\hline Tender abdomen & $27(50)$ & $27(50)$ & $54(55.1)$ & 0.025 \\
\hline Woody abdomen & $17(43)$ & $23(57)$ & $40(40.8)$ & 0.002 \\
\hline Palpable fetal parts & $40(73)$ & $15(27)$ & $55(56.1)$ & 0.003 \\
\hline Active bleeding & $11(48)$ & $12(52)$ & $23(23.5)$ & 0.166 \\
\hline Clots & $20(63)$ & $12(37)$ & $32(32.7)$ & 0.746 \\
\hline Metabolic acidosis & $24(51)$ & $23(49)$ & $47(48)$ & 0.076 \\
\hline Oxytocin augmentation & $10(37)$ & $17(63)$ & $27(27.6)$ & 0.004 \\
\hline Caesarean delivery & $40(66)$ & $21(34)$ & $61(62.2)$ & 0.163 \\
\hline Vaginal delivery & $19(51)$ & $18(49)$ & $37(37.8)$ & 0.216 \\
\hline
\end{tabular}


RESEARCH

Table 4. Maternal and fetal outcomes

\begin{tabular}{|c|c|c|c|}
\hline Variable & $<50 \%$ AP $(N=39), n(\%)$ & $>50 \%$ AP $(N=59), n(\%)$ & $p$-value \\
\hline \multicolumn{4}{|l|}{ Maternal } \\
\hline Thrombocytopaenia & $6(35)$ & $11(65)$ & 0.021 \\
\hline Disseminated intravascular coagulopathy & $0(0)$ & $1(100)$ & 0.326 \\
\hline Postpartum haemorrhage & $14(33)$ & $29(67)$ & $<0.001$ \\
\hline \multicolumn{4}{|l|}{ Fetal } \\
\hline Alive & $44(76)$ & $14(24)$ & $<0.001$ \\
\hline 5-minute APGAR score $>7$ & $36(82)$ & $8(18)$ & $<0.001$ \\
\hline Fresh stillbirth & $17(52)$ & $16(48)$ & 0.211 \\
\hline Macerated stillbirth & $1(11)$ & $8(89)$ & 0.003 \\
\hline
\end{tabular}

literature, a woody hard abdomen and difficulty in palpitating fetal parts are findings associated with a severe $\mathrm{AP}^{[1]}$ This was also the case in our study, thus highlighting the high index of suspicion for $\mathrm{AP}$ in pregnant women presenting with abdominal pain and vaginal bleeding, rather than the typically associated severe symptoms.

$\mathrm{CD}$ was the most common route of delivery as per guidelines, to ensure that delivery is carried out in the safest and quickest route, if the baby is alive. In our study, there was a higher number of live births than stillbirths, accounting for the higher caesarean delivery rate compared with vaginal deliveries. This finding is in keeping with a study by Akadri et al. ${ }^{[15]}$ in Nigeria.

The timeframe from presentation to delivery was $<3$ hours for the majority of the patients. A group in India ${ }^{[16]}$ found a mean duration of 5.2 hours in their study. Of the 6 maternal deaths in the aforementioned study, four were due to hypovolemic shock, one had disseminated intravascular coagulopathy and one died after eclampsia ${ }^{[16]}$ These deaths occurred within 2 - 4 hours of admission and the patients presented being as haemodynamically unstable. ${ }^{[16]}$ A shorter time interval to delivery forms part of the resuscitation process in patients who are haemodynamically unstable as this aborts the bleeding owing to $\mathrm{AP}$, allows effective volume expansion resuscitation and management of further postpartum haemorrhage $(\mathrm{PPH})$ can be executed more efficiently and definitively. ${ }^{[17]}$

The SA maternity care guidelines ${ }^{[18]}$ recommend immediate CD if vaginal delivery is not imminent if the baby is alive, if the mother is unstable or worsening organ dysfunction. The guidelines further recommend that if delivery is not likely to occur within 8 hours in a patient on oxytocin augmentation with ruptured membranes, a $\mathrm{CD}$ must be considered. Immediate delivery within 30 minutes in a patient with a live baby is often hampered by administrative issues such as transfer to an appropriate facility, theatre preparation and availability, and access to blood products.

Thrombocytopaenia was more common in the $>50 \%$ AP group. Johnson et al. ${ }^{[15]}$ found that $9.3 \%$ of their study population had thrombocytopenia associated with AP. This is due to consumption of platelets during haemostatic mechanisms activated by blood loss. Eight percent $(8 \%)$ of women had acute kidney injury (AKI) which is similar to the findings of Akadri et al. ${ }^{[15]}$ who reported that $12.2 \%$ of women had AKI, while Johnson et al. ${ }^{[20]}$ reported $1 \%$ with AKI in their study of 98 cases of AP. This difference may be due to multiple factors such as early resuscitation and mode of delivery, patients presenting earlier with milder forms of AP, or absence of comorbidities such as hypertension in pregnancy resulting is lower susceptibility to target organ dysfunction.
PPH was reported in $41 \%$ of the women in this study and was medically managed in most of the women. The occurrence of PPH was more frequent in women where the placental separation was greater than $50 \%$. Several studies have reported a high risk of PPH with $\mathrm{AP} \cdot{ }^{[15,16,19]}$ The likely cause of $\mathrm{PPH}$ is uterine atony as $33(75 \%)$ of the women with PPH were successfully managed with uterotonic agents. The reason for the atony, however, is not known, given that a Couvelaire uterus was not observed in these women. ${ }^{[1,11]}$

We found a stillbirth rate of 420/1 000 deliveries for our study population, compared with the national stillbirth rate of 25/1 000 deliveries. This difference highlights the high mortality associated with AP. AP was the third most common cause of perinatal mortality in a study in Limpopo Province, SA. ${ }^{[19]}$

Our study highlights the unpredictability of AP. A majority of patients who have AP do not have the well-documented risk factors of AP. The classical signs of AP are significantly more frequent in those with severe AP. Our study findings support the recommendation of early resuscitation and quicker delivery to prevent complications of AP. The strengths of the study are the prospective design that allowed collection of real-time data. All patients who presented with AP consented to participation in the study; it is therefore a true reflection of women managed at the two institutions. Among the limitations were that that there was no control group to evaluate the association between risk factors and outcome. Furthermore, the sample size of the study population was small.

\section{Conclusions}

In our setting, AP is a condition present in $\sim 1 \%$ of all maternal deliveries. We have shown that it is highly unpredictable as the known risk factors are not common in those presenting with the condition. The symptoms and signs of abruptio placentae are often late presentation features. Although AP poses significant perinatal and maternal morbidity and mortality, early interventions can prevent poor maternal and neonatal outcomes.

\section{Acknowledgements. None.}

Author contributions. BM and SA developed the research protocol as part of a Master's degree. BM was responsible for data collection and management and SA supervised the study. TN was responsible for statistical analysis. SA and BM prepared the manuscript for submission.

Funding. None.

Conflicts of interest. None. 


\section{RESEARCH}

1. Buchman EJ. Antepartum haemorrhage. In: Cronje HS, Grobler CJF, eds. Obstetrics in Southern Africa. Cape Town: Van Schaik Publishers, 202-209.

2. Ananth CV. Placental abruption and its association with hypertension and prolonged rupture of membranes: A methodologic review and meta-analysis. Obstet Gynecol 1996;88(2):309-318. https://doi.org/10.1016/0029-7844(96)00088-9

3. Ananth CV, Wilcox AJ. Placental abruption and perinatal mortality in the United States. Am J Epidemiol 2001:153(4):332-337. https://doi.org/10.1093/aje/153.4.332

4. South African National Department of Health. Saving Mothers 2014 - 2016: Seventh triennial repor on confidential enquiries into maternal deaths in South Africa: Short report. https://www.sasog. co.za/Content/Docs/Saving_Mothers.pdf. (Accessed 30 July 2019)

5. Ananth CV, Lavery JA, Vintzileos AM, et al. Severe placental abruption: Clinical definition an associations with maternal complications. Am J Obstet Gynecol 2016;214(2):272.e1-272.e9. https:/ doi.org/10.1016/j.ajog.2015.09.069

6. Mukheriee S. Bawa AK Sharma S, Nandanwar YS, Gadam M. Retrospective study of risk factors and 6. Muthe $S$, Ben maternal and fetal outcome in patients with abruptio $p$

7. Jabeen M, Gul F. Abruptio placentae: Risk factors and perinatal outcome. J Postgrad Med Ins 2004;18(4):669-676.

8. Boisramé T, Sananès N, Fritz G, et al. Placental abruption: Risk factors, management and maternalfetal prognosis. Cohort study over 10 years. Eur J Obstet Gynecol Reprod Biol 2014;179:100-104. https://doi.org/10.1016/j.ejogrb.2014.05.026

9. Dars S, Sultana F, Akhter N. Abruptio placentae: Risk factors and maternal outcomes at a tertiary care hospital. JLUMHS 2013;12(3):198-202. https://www.lumhs.edu.pk/jlumhs/Vol12No03/pdfs/14 pdf (accessed 30 July 2019)

10. Ananth CV, Getahun D, Peltier MR, Smulian JC. Placental abruptio in term and preterm gestations: Evidence for heterogeneity in clinical pathways. Obstet Gynaecol 2006;107(4):785-792. https://doi. Evidence for heterogeneity in clinical path
11. Hall DR. Abruptio placentae and disseminated intravascular coagulopathy. Semin Perinatol 2009;33(3):189-195. https://doi.org/10.1053/j.semperi.2009.02.005

12. Brink AL, Odendaal HJ. Risk factors for abruptio placentae. S Afr Med J 1987;72(4):250-252.

13. Anakiram P, Sellathamry SG, Ponnivalavan K. Maternal and perinatal outcome in abruptio placenta. J Evid Based Med Healthc 2017;4(67):3985-3988. https://doi.org/10.18410/jebmh/2017/797

14. Tikkanen M. Etiology, Clinical manifestations, and prediction of placental abruption. Acta Obstet Gynecol Scand 2010; 89(6):732-740. https://doi.org/10.3109/00016341003686081

15. Akadri AA, Ogunsowo KM, Odelola OI. Abruptio pacenta: A retrospective analysis in a tertiary hospital, Sagamu, Nigeria. Trop J Obstet Gynaecol 2018;35(2):142-146. https://doi.org/10.4103/ TJOG.TJOG_14_18

16. Renuka P, Kumari A, Akhila K. Maternal and perinatal outcome in abruptio placenta - study at teaching hospital. IAIM 2016;3(10):111-116. http://iaimjournal.com/wpcontent/uploads/2016/10/ iaim_2016_0310_18.pdf (accessed on 20 August 2019)

17. Oyelese Y, Ananth CV. Placental abruptio. Obstet Gynaecol 2006;108(4):1005-1016. https://do org/10.1097/01.AOG.0000239439.04364.9a

18. National Department of Health South Africa. Guidelines for Maternity Care in South Africa. A Manual for Clinics, Community Health Centres and District Hospitals. Pretoria: NDoH, 2015.

19. Ntuli ST, Malangu N. An investigation of the stillbirths at a tertiary hospital in Limpopo province of South Africa. Glob J Health Sci 2012;4(6):141-147. https://doi.org/10.5539/gjhs.v4n6p141

20. Johnson JK, Mukhopadhay M, Ray N, Lindow SW. Abruptio placenta-a retrospective study of 98 cases. J Obstet Gyn 2003;23(Suppl1):S70-S70. https://doi.org/10.1080/71859170

Accepted 12 January 2020 